\title{
The Development of the Automatic Pick-and-Placing Manipulator and Control System
}

\author{
Du Xueliang ${ }^{1, a}$, Yin Mingfu ${ }^{2, b}$ \\ ${ }^{1}$ Tianjin Polytechnic University, Tianjin 300387, china \\ ${ }^{2}$ Tianjin Polytechnic University, Tianjin 300387, china \\ a563834487@qq.com, byinmingfu@163.com
}

Keywords: manipulator, 3D model, force/position control, fuzzy adaptive control

\begin{abstract}
At present, most of the factories carry materials by hand about ceramic. This paper designed the structure of the product with the automatic pick-and-placing manipulator as the research object. Furthermore, it applied 3D model and motion simulation to the product through SolidWorks. Then it gave a design scheme of the manipulator. Finally it obtained the feasibility of the design scheme. At the same time, this paper adopted the control strategy with force ring and position ring and fuzzy control adoptive PID algorithm to ensure the precision requirement. It also made a dynamic model of the manipulator structure for the movement of the manipulator. The control strategy can achieve good control effects through the analysis by MATLAB simulation.
\end{abstract}

\section{Introduction}

At present, Pick-and-Placing supplies is still on the manual stage in the production process of the ceramic in our country. On abroad, it has made significant progress to industrial robot and manipulator, implemented the intelligent of the manipulator ${ }^{[1]}$. The industry manipulator has been preliminary application in our country, our industrial manipulator on the whole has a larger difference with foreign developed countries both in the technical level, scale of development and product performance. Therefore, the papers has deeply researched and innovated the structure and control system of the pick and place manipulator in the process of ceramic and designs an automatic Pick-and-Placing manipulator for the domestic small and medium-sized enterprise.

\section{The design of the manipulator structure}

According to the actual requirement of the enterprises, when we design the structure of the Pick-and-Placing manipulator, it not only takes a full consideration about working conditions, cost, and function, but also has the characteristics of the easy operation and service, high process efficiency. The manipulator is made up of the foundation, translator unit, Pick-and-Placing unit, etc. The manipulator moves as follows basis on the requirements of the production line:

(1) $\mathrm{X}$ moving range: $0 \sim 500 \mathrm{~mm}$

(2) Y moving range: $0 \sim 200 \mathrm{~mm}$

(3) Y rotation angle range: $0 \sim 5^{\circ}$

(4) $Z$ rotation angle range: $0 \sim 90^{\circ}$

The design of translator unit. The translator unit uses servo motor driving, gear-rack transmission, and the gantry structure. It has not only reducing the moving mass and increasing the translator part stiffness but also having the characteristic of easy operation and full-featured. The servo motor adopt the fifth generation SGMJV series of 17 encoder servo motor and servo drives about YASKAWA. It can ensure the accuracy requirement of $\mathrm{X}$ and $\mathrm{Y}$ with high running precision and stable operation.

The design of pick-and-placing unit. It using the structure of worm gear and bevel gear associated operation by the servo motor. The picking hand can adopt different structure according to the material shape. As shown in Fig.1, the wording principle of the manipulator is as follows: The picking hand is driven by servo motor 4 and 10,and the bevel gear. Then Y axis rotates $5^{\circ}$ and holds up the ceramics. And then the servo motor 6 drives worm gear, so the picking hand rotates along $\mathrm{Z}$ 
to arrive at the designated spot.

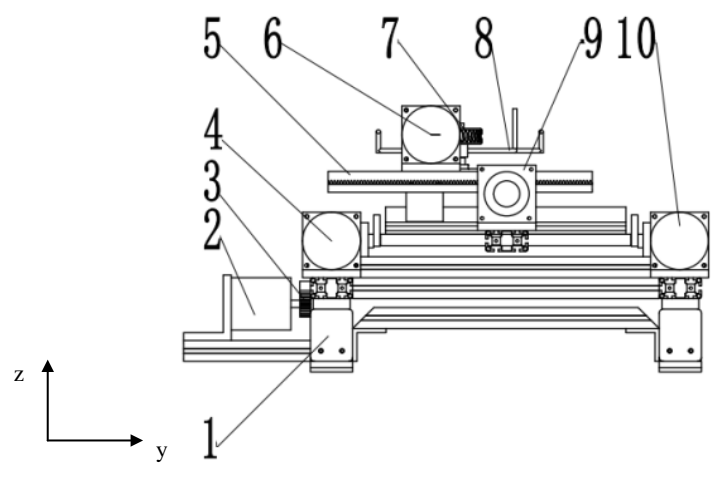

Fig.1 Picking manipulator left view

3D model and simulation built about manipulator. The paper establishes 3D model about manipulator by SolidWorks (Fig.2) and assembles it according to the process. And it checks the interference problems in the movement process by SolidWorks motion simulation. The time control diagram of the manipulator simulation is as shown in Fig.3:

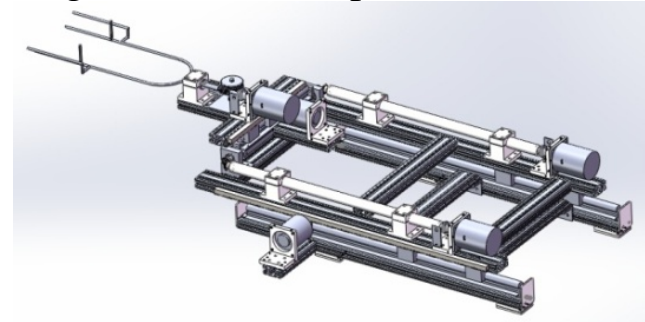

Fig.2 Manipulator 3D model

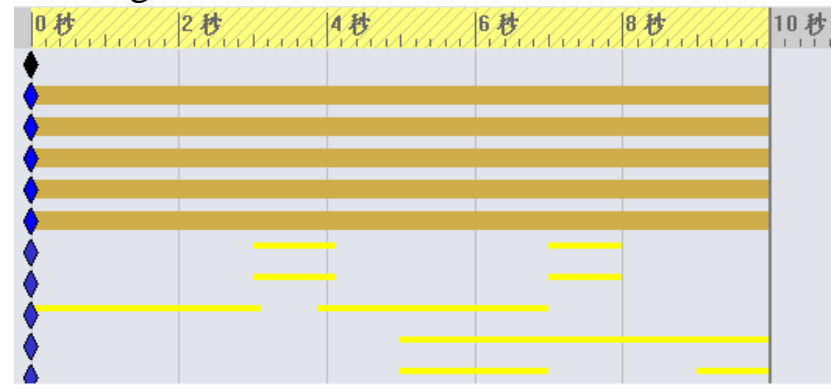

Fig.3 Manipulator movement time diagram

\section{The design of motion control system}

The control system of hardware parts. In this paper, The parts of control system hardware consists of EVOC, motion control card, servo motor and the related drive, electrical control cabinet, sensors etc. The paper adopts the currently popular structure of open control system, with DSP as the core processor ${ }^{[2]}$, which is based on the PCI bus and uses PC as information processing platform. Motion controller in the form of card embedded PC, as same as PC + motion controller model. It chooses the MPC08SP four shaft motion control card made by Lechuang Automation Co. Ltd as the motion control cards. It chooses the 1051 type piezoelectric force sensor made by Dytran company as the pressure sensor. EVOC as the host computer, based on the VC ++ to complete the control system of human-computer interaction, management and coordination of various modules. The hardware structure of PC + motion control card shown in fig.4: 


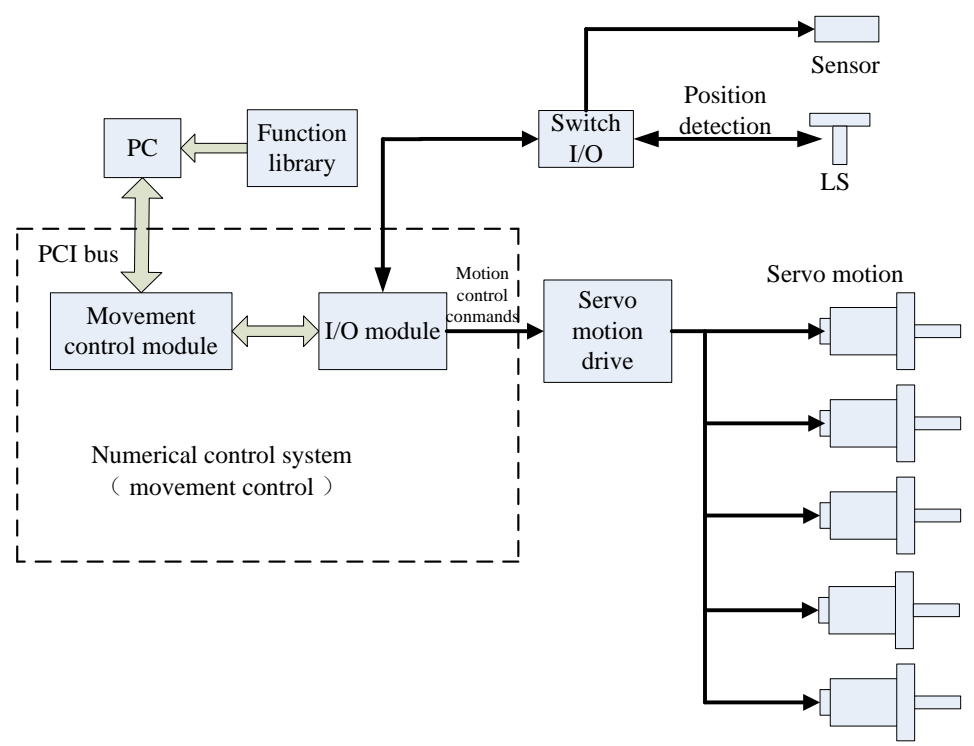

Fig.4 The control system diagram

Manipulator for feeding process analysis. Specifically manipulator brings the ceramics from furnace mode to the specific location on the next working procedure, and the whole movement is divided into forward, pick up, transfer, put-down, and back to the original, as shown in fig.5:

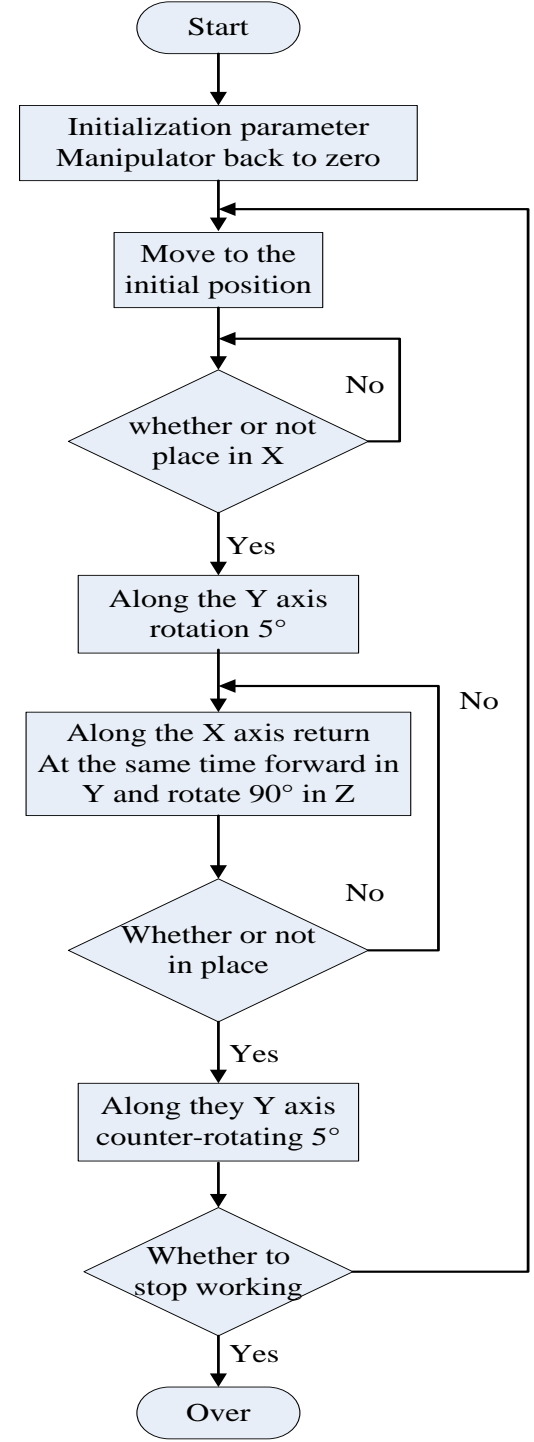

Fig.5: the manipulator motion sequence diagram

Manipulator for feeding process model. According to the analysis of reclaimer and blowing process by the manipulator in 3.1, the system model of the process can be divided 
into two parts. One is the servo feed system model of the manipulator. The other part is the sensor model mounted on the manipulator.

The manipulator is composed of servo motion, coupler, gear rack, bearing and the movement ${ }^{[3]}$. The dynamics model of the manipulator is as shown in fig.6.

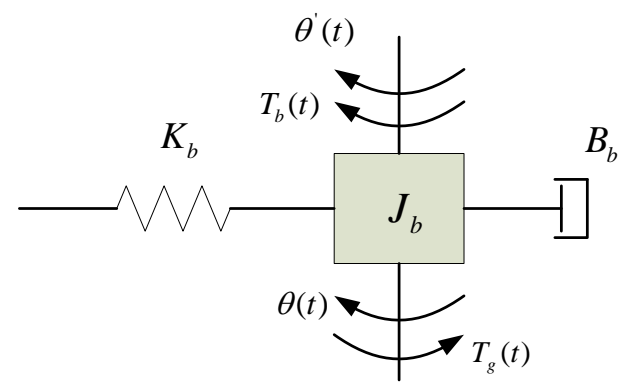

Fig.6 The equivalent dynamics model of manipulator

It can obtain the transmission balance equation of the manipulator by figure6.

$$
J_{b} \frac{d^{2} \theta(t)}{d t^{2}} R^{2}+R B_{b} \frac{d \theta(t)}{d t}+T_{g}(t)=T_{b}(t)
$$

Among them, $\theta(t)$ is the output angular of the gear and rack, $T_{b}(t)$ is the driving torque of the gear rack, $J_{b}$ is the equivalent inertia of gear rack, $B_{b}$ is the equivalent damping coefficient of gear rack, $T_{g}$ is the interference torque. Within the scope of the elastic deformation of gear rack, the drive torque is as follows:

$$
T_{b}(t)=R K_{b}\left[\theta^{\prime}(t)-\theta(t)\right]
$$

$\theta^{\prime}(t)$ is input angular of the gear and rack, $K_{b}$ is the equivalent stiffness of gear rack. Making the (3.2) and (3.1) to the Laplace transform and simplification, it can obtain as follows:

$$
\theta(s)=\frac{K_{b} \theta^{\prime}(s)-T_{g}(s) / R}{J_{b} R s^{2}+B_{b} s+K_{b}}
$$

When it is $T_{g}(s)=0$, the manipulator transfer function between input and output is as follows:

$$
G_{m}(s)=\frac{\theta(s)}{\theta^{\prime}(s)}=\frac{K_{b}}{J_{b} R S^{2}+B_{b} s+K_{b}}
$$

The control strategy and method in the process of feeding. The control system uses different control strategies aiming at different stages of movement. In fast approaching and high-speed returning stage, it just need high precision position control by the manipulator. When in the accurate put stage, it can take the output of the force loop controller as the location of the position of loop controller to complete the compensation input. Namely, the external loop and the position inner loop control together. The control system structure of the movement process for manipulator is shown in fig.7:

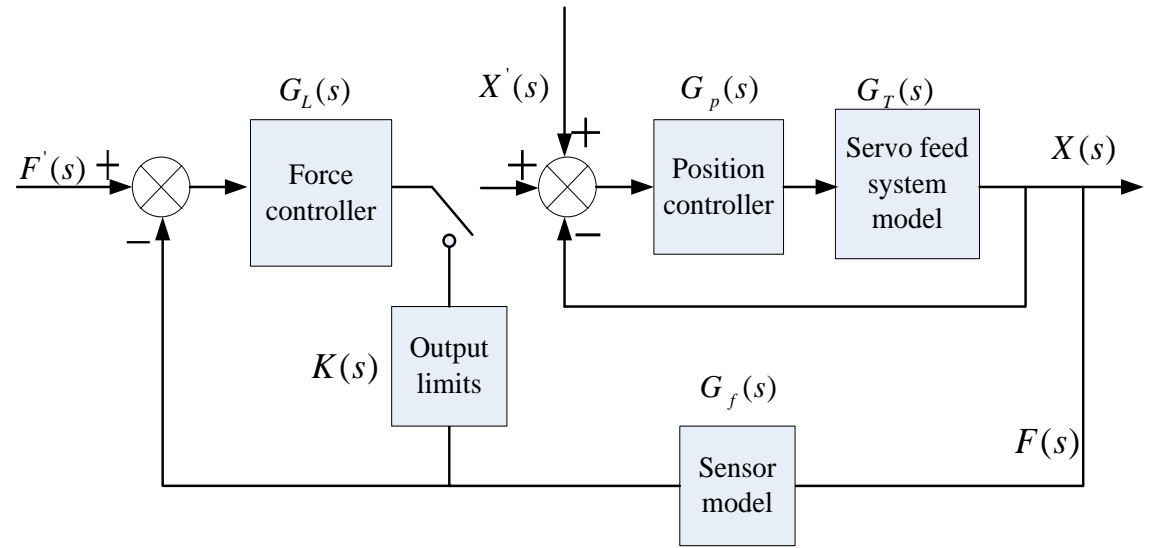

Fig.7 The control system of manipulator structure 
The transfer function of control system about the whole manipulator is as follows $\left(k_{1}, k_{2}\right.$ is the coefficient):

$$
\left(F^{\prime}(s)-F(s)\right) G_{L}(s) K(s) G_{T}(s) G_{f}(s)+\left(X^{\prime}(s)-X(s)\right) G_{p}(s) G_{T}(s)=k_{1} X(s)+k_{2} F(s)
$$

\section{The fuzzy adaptive control system}

The design of the fuzzy adaptive control system. The fuzzy adaptive PID control is a kind of method combining traditional PID control and fuzzy control. In view of the manipulator picking material in the process of movement, it takes the deviation of contact force $e(t)=f_{0}(t)-f_{i}(t)$ and the deviation rate of change $e_{c}(t)=\frac{d e(t)}{d t}$ as the input of the fuzzy adaptive control ${ }^{[5]}$. As the figure 4-1 shown, the $x_{p}, x_{i}, x_{d}$ is the modification coefficient of $K_{p}, K_{i}, K_{d}$ about PID parameters. The membership function is Gaussian function: $\mu_{i}\left(A_{i}\right)=\exp \left(\frac{A_{i}-0.2+i}{3}\right)^{2}$

Among them, $A_{i}=\left[e_{i}, e_{c i}, x_{p}, x_{i}, x_{d}\right]$

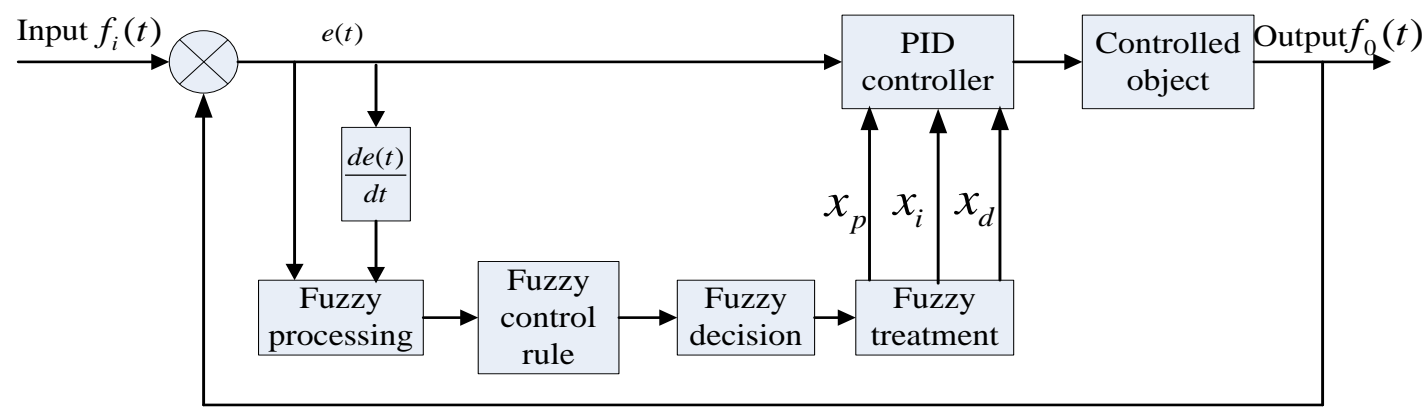

Fig.8 Fuzzy self-adaptive control principle diagram

The realization of fuzzy controller. Through the MATLAB simulation function, according to the principle of the fuzzy control in figure 4.1, it generates the output force curve, as show in fig.9.

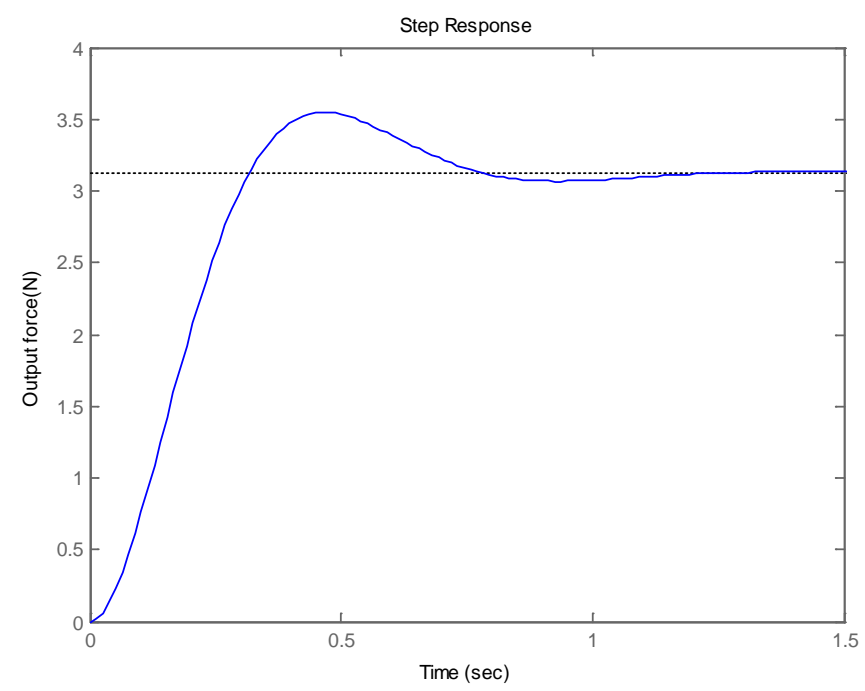

Fig.9 Output curve of the fuzzy adaptive

\section{Conclusion}

This topic researches automatic feeding manipulator which is used to Pick-and-Placing materials. The control system model is set up with SolidWorks and motion simulation, as well as through analyzing the dynamic model of machinery. Furthermore, it uses the "external ring and position 
inner loop" control strategy and control method of fuzzy adaptive controller, and develops the control system of the Pick-and-Placing manipulator which is based on MPC08SP.

\section{Reference}

[1] He Chongdong. Control System Design and Implementation of Robotic Manipulators for Pick-and-Placing GDL [D]. Huazhong University of Science and Technology.2013

[2] Li xinjian. A Design of Motion Controller for Multi-axes Based on PCI Bus. Nanjin University of Aeronautics and Astronautics .2007

[3] Wang Hui. The Analysis and Simulation of AC Servo Feeding System of CK6136 NC Lathe [D].Master's Thesis, Zhejiang University of Technology , 2009.

[4] Li Xiaoyan. Research and Application on Force Position Control of Robot[D]. Master's Thesis, Hebei University,2011.

[5] Qiao Zhijie, Wang Weiqing. The Design of The Fuzzy Adaptive Controller and its Computer Simulation [J]. Automation and Instrumentation ,2008,(01) 